\title{
Microanalysis of Cd Whiskers on Cd Plated Long-Term Used Hardware
}

Sara Dickens ${ }^{1}$, Tim Ruggles ${ }^{1}$, Rachel White ${ }^{2}$, Zahra Ghanbari ${ }^{2}$, Daniel Perry ${ }^{2}$ and Donald Susan ${ }^{2}$

${ }^{1}$ Sandia National Laboratories, Albuquerque, New Mexico, United States, ${ }^{2}$ Sandia National Laboratories, United States

Cadmium whiskers were recently observed on field-return hardware at Sandia during a fastener failure analysis study unrelated to whisker growth-In this study, approximately 200 cadmium plated cap screws and 400 cadmium plated nuts with varying service lifetimes, were examined and cadmium whisker growth was ubiquitous. Previously published characterization work on cadmium whiskers contained smaller samples of whiskers and relied on whiskers grown from the vapor phase. These studies were also conducted prior to the advent of modern EBSD technology [1,2]. Here we report on the microanalysis of cadmium whisker growth that occurred organically on cadmium plated hardware. They demonstrated a preferred growth direction of $\langle-12-10\rangle$, but begged the question- is this direction also preferred by whiskers grown from solid state plating? Why is direction preferred? What other growth directions are possible? These questions were addressed by characterizing 50 cadmium whiskers with scanning electron microscopy (SEM) and electron backscatter diffraction (EBSD).

The cadmium whiskers were washed from the fasteners using alcohol for transfer to aluminum stubs, resulting in minimal distortion of the whiskers. A Zeiss Supra 55-VP Field Emission Scanning Electron Microscope equipped with an Oxford Symmetry CMOS Electron Backscatter Detector was utilized for data collection. The hardware with the most wear was observed to contain the highest density of whiskers. The whiskers exhibited various morphologies- nodes/nodules, straight, kinked, and curled. These whiskers were observed to exist both alone and in agglomerates. These whiskers underwent no surface preparation.

EBSD analysis determined a strong preferred growth direction of $\langle-12-10\rangle$, consistent with studies conducted on whiskers grown in the vapor phase. The 50 whiskers selected were straight to minimize ambiguity in the growth direction. Of these 50 whiskers, $80 \%$ fell within $15^{\circ}$ of the $\langle-12-10\rangle$ growth type direction. The remaining $20 \%$ exhibited random growth directions. This is illustrated in the misorientation histogram in Figure 1. Focused Ion Beam (FIB) cross sections of six whiskers were done in order to determine which planes parallel to $\langle-12-10\rangle$ type growth direction comprised the side faces. One of these cross sectioned whiskers is exhibited in Figure 2. EBSD of the whisker cross sections determined that (0001) and \{10-10\} low energy side faces comprised 52\% of the surface area of the whiskers examined. This suggests that, although whisker growth produces new surface area, the low energy surfaces help minimize the overall energy of the system. 


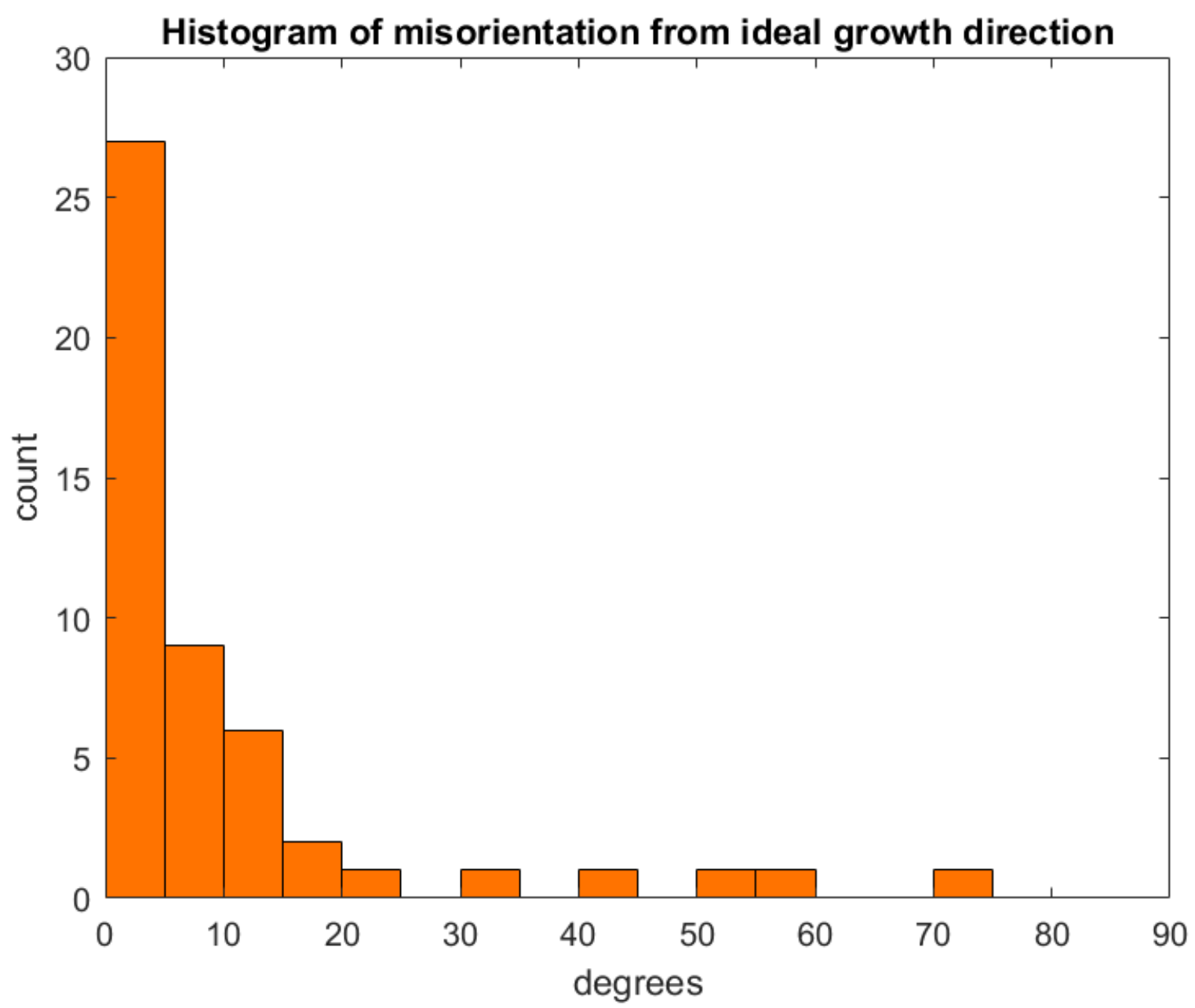

Figure 1. Histogram representation of misorientation from the ideal $<-12-10>$ growth direction

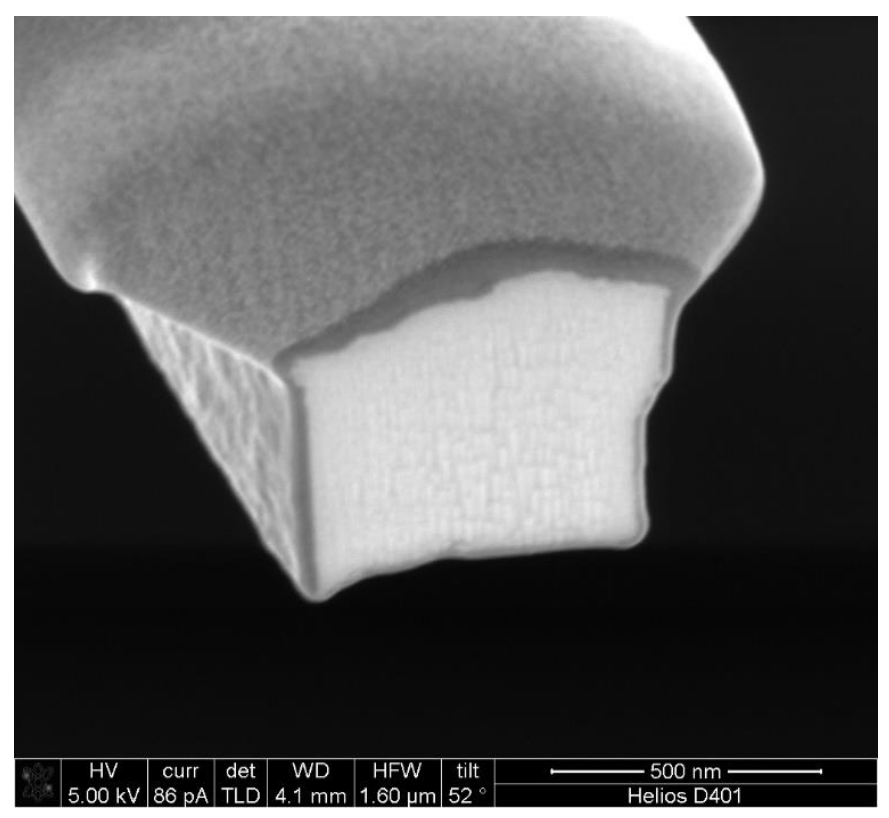


Figure 2. FIB cross section of a cadmium whisker. Cover layer is Pt to protect the whisker during FIB cutting.

\section{References}

[1] Nanev, C. and D. Iwanov, Growth of Zinc and Cadmium Whiskers.Physica Status Solidi, 1967. 23: p. 663-673.

[2] Verma, A.R. and S.K. Peneva, X-ray Investigation on the Growth of Cd Whiskers. Journal of Crystal Growth, 1968. 3: p. 700-704.

This paper describes objective technical results and analysis. Any subjective views or opinions that might be expressed in the paper do not necessarily represent the views of the U.S. Department of Energy or the United States Government. Sandia National Laboratories is a multimission laboratory managed and operated by National Technology and Engineering Solutions of Sandia, LLC., a wholly owned subsidiary of Honeywell International, Inc., for the U.S. Department of Energy's National Nuclear Security Administration under contract DE-NA-0003525

SAND2021-2100 